International Journal of Biomedicine | June 2021 - Volume 11, Issue Suppl_1: Abstracts from the Third Russian International Conference "Cryo-electron microscopy 2021: achievements and prospects"

POSTER ABSTRACT PRESENTATIONS

SESSION TITLE: EM RESEARCH RELATED TO MEDICINE

DOI: 10.21103/IJBM.11.Suppl_1.P32

\title{
Abstract P-32: Lung Structural Changes in Covid-19 Patients
}

$\underline{\text { Valeriy P. Kartashev }}^{1}$, Djamshid N. Mardonov ${ }^{2}$, Bahshillo R. Mamirov ${ }^{3}$, Azamat Kh. Butaev ${ }^{3}$

${ }^{1}$ Russian State Social University, Moscow, Russia

${ }^{2}$ Republican Specialized Scientific Practical Medical Center of Surgery named after acad. V.Vakhidov, Tashkent, Uzbekistan

${ }^{3}$ First Zangiota Specialized Infectious Diseases Hospital, Tashkent, Uzbekistan

Background: The main causes of death from Covid-19 are lung lesions with the development of respiratory failure. However, structural changes in the lung tissue in this pathology are poorly studied. We examined autopsy material from patients with Covid-19.

The severe condition of patients, the manifestation of pulmonary symptoms of damage (cough, dyspnea) and the high probability of viral pneumonia at COVID-19 led to the widespread use of CT diagnostics in this group of patients, which allowed to identify of the primary signs of the disease, their subsequent transformation as well as the most adverse radiation symptoms corresponding to the severe course of the process (Speranskaya, 2020; Pan et al., 2019).

In the cases studied by us, the detection of typical symptoms revealed by radiation diagnostics of COVID-19 was subsequently confirmed by PCR data, which may indicate a high information content and specificity of detecting CT symptoms of a lesion as a method of primary diagnostics.

The aim of our study is to confirm at the microscopic level the correspondence of MSCT changes.

Methods: The material was taken at autopsy of deceased patients, fixed in $10 \%$ formalin solution in phosphate buffer, paraffin sections were stained with hematoxylin and eosin. The autopsy material was examined using a Carl Zeiss light microscope, Axioskop 40.

Results: Studies have shown that most of the alveoli of the lung tissue kept their airiness. However, their lumens were significantly reduced due to a significant thickening of the interalveolar septa caused by pronounced inflammatory 
infiltration mainly by lymphocytes. The most significant changes were revealed from the side of the microvasculature. There are numerous blood clots of various sizes in the lumen of most micro-vessels. The walls of microvessels have been significantly thickened with pronounced inflammatory infiltration and significant edema. The lumens of microvessels have been characterized by significant polymorphism. Intraluminal clots have been also characterized by pronounced polymorphism. The parietal pleura has been thickened. This has been reflected in the MSCT images and corresponds to the processes of perivascular infiltration.

Conclusion: The primary CT pattern of COVID-19 is a picture of infiltration of individual secondary pulmonary lobules of the "ground glass" type, followed by a decrease in the lesion volume at a favorable course of the disease, or their increase, the addition of a CT picture of a "cobblestone pavement" and the appearance of alveolar infiltration in the area of "ground glass" at the unfavorable course of the disease.

Key Words: COVID-19 $\bullet$ pneumonia $\bullet$ lymphocytes $\bullet$ microvessels

*Corresponding author: Valery Kartashev.E-mail: kvpmos@mail.ru

International Journal of Biomedicine. 2021;11 Suppl 1: S25-26.

doi: 10.21103/IJBM.11.Suppl_1.P32

(C)2021 International Medical Research and Development Corporation 